\title{
Delirium, a 'confusing' condition in general hospitals: The experience of a Consultation- Liaison Psychiatry Unit in Greece
}

This article was published in the following Dove Press journal:

International Journal of General Medicine

14 October 2009

Number of times this article has been viewed

\author{
Panagiota Goulia \\ Christos Mantas \\ Thomas Hyphantis \\ Consultation-Liaison Psychiatry Unit, \\ Department of Psychiatry, Medical \\ School, University of loannina, \\ loannina, Greece
}

Background/aims: A plethora of studies showed that delirium is common in hospitalized populations. We aimed to examine the characteristics of delirium patients referred to our Consultation-Liaison Psychiatry Unit (CLPU).

Methods: Our CLPU database was used to obtain data of all referred patients admitted to our hospital and diagnosed with delirium. All referred nondelirious patients served as controls.

Results: During one year, 483 patients were referred to the CLPU. Ninety-three (19.3\%) were diagnosed with delirium. Delirious patients were older than nondelirious patients $(P<0.001)$, with $76.3 \%$ aged over 70 years. The majority of the referrals came from surgical specialties. Common etiological factors were fluid and electrolyte imbalance (29\%), fractures (28\%) and infections (24\%), but laboratory tests for the investigation of the etiology prior to the consultation had been performed in only 12 patients (12.9\%). The syndrome resulted in prolonged hospitalization and greater use of CLPU services.

Conclusions: Referrals for delirium are frequent in CLPUs in Greece. Although delirium is common, it remains a 'confusing' condition for health practitioners. The under-diagnosis of delirium, the prolonged hospitalization and the time that the CLPU dedicated to these patients underlines the role of the CLPU psychiatrists in the management of the syndrome.

Keywords: delirium, general hospital, consultation-liaison psychiatry, referrals, Greece

\section{Introduction}

Delirium, the Cinderella of medicine, as aptly coined by Leentjens and colleagues ${ }^{1}$ afflicts patients, relatives and nurses, troubles physicians, and consumes much of the Consultation-Liaison Psychiatry Unit (CLPU) psychiatrists' time. Delirium is defined as an acute change in cognition and a disturbance of consciousness with impaired attention that fluctuates during the course of the day. ${ }^{2}$ It is a frequent condition in general hospitals with a high prevalence on admission $(11 \%-33 \%)^{3-6}$ and incidence during hospital stay $(3 \%-56 \%) ., 4,7,8$ It is associated with increased morbidity and mortality, greater use of hospital resources, longer hospital stays, and increased rates of nursing home placement on discharge..$^{9-12}$

Despite these facts, the recognition rates are $l_{0} \mathrm{w}^{5,13-15}$ and the management of delirium remains inadequate in up to $80 \%$ of patients, ${ }^{13}$ suggesting a lack of preventive measures and screening tests, missed diagnoses, and inappropriate management of diagnosed delirium. ${ }^{6}$ Furthermore, although evidence-based guidelines are increasingly being developed by professional psychiatric organizations in an attempt to improve clinical practice, the national psychiatric associations of only two countries have such a guideline for the diagnosis and treatment of delirium. ${ }^{16}$
Correspondence: Panagiota Goulia

Department of Psychiatry, Medical School,

University of loannina, loannina $45 \mathrm{I}$ I0,

Greece

Tel +302651097255

Email panagiotagoulia@yahoo.com 
It has also been suggested that by identifying the specific characteristics of delirium patients who are referred to a CLPU service, psychiatrists may make targeted efforts to educate primary providers about detection and referral for vulnerable populations. ${ }^{17}$ This would give providers additional incentive for detecting and referring delirium patients, and provide CLPU psychiatrists even stronger justification for the utilization of their services. ${ }^{17}$

Although the syndrome is known since the Hippocratic era (circa $400 \mathrm{BC})^{18}$ research on delirium in Greece is scarce. ${ }^{19,20}$ Furthermore, to the best of our knowledge, no studies have investigated the frequency and clinical characteristics of delirium in general hospitals in Greece. Prompted by this fact, the aim of the present study was to examine the frequency, clinical profile and management of delirium in a tertiary general hospital in Greece during a period of one year, in the context of the recently established CLPU of the Department of Psychiatry, University of Ioannina, Greece.

\section{Patients and methods Setting}

The study was carried out at the University General Hospital of Ioannina, Greece, which is a tertiary teaching hospital with 850 beds, providing secondary and tertiary care for a general population of 350,000. In December 2006, an independent CLPU was established. ${ }^{21}$ The CLPU staff consists of two full-time and one part-time consultant psychiatrists, two full-time residents in psychiatry, one full-time and one part-time clinical psychologist, four $\mathrm{PhD}$ students, and four undergraduate medical students. The unit covers the inpatients in the hospital. The Accident and Emergency Department is covered by the Psychiatric Department's 24-hour service.

Inpatients are assessed within 24 hours from the referral (unless there is an emergency situation) after a meeting with the patient's physician, a nurse, and a patient's family member (if available) has been held. After assessment, a brief psychiatric impression and advice is written in the patient's medical record, and a written psychiatric report is given to the physician. Details of patients' referral to the CPLU are recorded and entered into our electronic database, developed especially for the unit. This includes information on the following: demographics; reason and source of the referral; people interviewed; days of hospitalization; brief description of the patient's current state; medical, surgery, and medication history; family history; detailed psychiatric history; developmental and social history; legal problems; relevant life events; current physical examination; current laboratory findings; present mental state examination; and diagnostic impressions according to Diagnostic and Statistical Manual of Mental Disorders, fourth edition (DSM-IV) criteria (all axes including global assessment of functioning [GAF]).

From the database an answer sheet is printed from a psychiatric consultation note immediately after the data has been keyed in, which includes the main findings of the psychiatric assessment, our diagnostic impression and suggestions, a brief therapeutic plan, our recommendations regarding the patient's capacity or suicidality, goals, objectives, and risks of the suggested therapy, together with any possible side effects of the suggested psychopharmacological agents. During hospitalization, there are regular follow-ups and, after discharge, patients are referred to our unit's services for the continuity of their care.

The management of the patients with delirium includes the following: The relief of the symptoms, the identification and treatment of the putative etiological factor(s) and the prevention of physical damage to the patient or others, which are accomplished by suggesting laboratory exams when appropriate and implementing pharmacological, environmental and supportive interventions. The latter two include: reassurance and information, reorientation, environmental modification (to ensure adequate sleep and appropriate stimulation), and the reduction of sensory deficits. During hospitalization there are regular follow-ups by the same CLPU staff member(s) and a follow-up appointment after discharge is programmed either with our service or with the other services of the local community mental health network, if needed.

\section{Design}

The study covers the period January 2007-December 2007. The data were obtained from the CLPU database. All the patients that were diagnosed with delirium were included and their data were compared to the data of all the patients that were assessed by the CLPU during the same period. For calculating the frequency rates, the hospital statistics data for the corresponding period were used. All the statistical analyses were performed using the Statistical Package for the Social Sciences for Windows (SPSS, v 15.0; SPSS Inc., Chicago, IL, USA). Summary statistics for all variables were calculated and two-tailed t-tests or chi-square tests were used, as appropriate..$^{22}$ All the procedures followed were in accordance with the ethical standards on human experimentation from the Declaration of Helsinki and were approved by 
the ethical committee of the Ioannina University General Hospital.

\section{Results}

\section{Referrals, diagnoses, and rates}

During a period of 12 months, 48,244 patients were admitted to our hospital (with the exclusion of psychiatric, pediatrics, neonatal and child surgery units because the first is served by its own staff and the latter three by the child psychiatrists). Of these, 483 were referred to the CLPU. Consequently, the annual psychiatric referral rate was $1 \%$.

The reason for the referral in 113 patients $(23.4 \%)$ was the acute onset of a combination of the following symptoms: confusion (43.2\%), agitation (18.9\%), hallucinations (32.4\%), delusions $(16.2 \%)$, disorientation $(72.9 \%)$, or insomnia $(62.1 \%)$. The mean duration $( \pm \mathrm{SD})$ of the symptoms was $2.00 \pm 0.38$ days prior to the request for consultation (median, two days). Ninety-three of these 113 referred patients (19.2\% of the total referrals) were eventually diagnosed with delirium by the CLPU staff.

In nine of the 113 cases, no certain diagnosis could be made because the referral was delayed and the symptoms subsided. The retrospective information from the staff, the relatives and the patients' charts led to the conclusion that seven patients had developed delirium. All were aged over 80 years and hypoactive. Five had a pre-existing history of dementia. The patients had developed the symptoms 3-5 days prior to consultation. Four of them had been administered haloperidol by their physicians prior to the referral. In the remaining three, the symptoms subsided without any intervention.

In 11 of the 113 cases, although the patients were described as delirious the final diagnosis was different (ie, dementia, psychosis, depression or behavioral problems attributed to the underlying physical disease). The patients were mainly agitated; some of them manifested common symptoms shared by psychiatric disorders including delirium (ie, delusions, hallucinations, insomnia) but not confusion, which is the hallmark of delirium.

\section{Patients' profile}

The main characteristics of delirious and nondelirious groups are shown in Table 1. As shown in this table, delirious patients were older than nondelirious patients $(P<0.001)$ with $76.3 \%$ aged over 70 years compared to $25.6 \%$ of the nondelirious group $(P<0.0005)$. In addition, $60.2 \%$ of delirious patients were male, in comparison with $46.1 \%$ of nondelirious group $(P<0.01)$. Delirious patients were mostly referred by the
Table I Patients' profile

\begin{tabular}{llll}
\hline & $\begin{array}{l}\text { Delirious } \\
\text { patients } \\
(\mathbf{n}=\mathbf{9 3})\end{array}$ & $\begin{array}{l}\text { Nondelirious } \\
\text { patients } \\
(\mathbf{n}=\mathbf{3 9 0})\end{array}$ & P-value \\
\hline $\begin{array}{l}\text { Sex } \\
\quad \text { Male }\end{array}$ & $56(60.2 \%)$ & $180(46.1 \%)$ & $P<0.0 I^{\prime}$ \\
$\quad$ Female & $37(39.9 \%)$ & $210(53.8 \%)$ & \\
Age (mean \pm SD) & $74.0 \pm 12.9$ & $55.3 \pm 20.3$ & $P<0.00 I^{2}$ \\
Age $>70$ years & $73(76.3 \%)$ & $100(25.6 \%)$ & $P<0.0005^{\prime}$ \\
Number of evaluations & $3.13 \pm 1.32$ & $1.96 \pm 0.76$ & $P<0.00 I^{2}$ \\
(mean \pm SD) & $9(9.7 \%)$ & $28(7.2 \%)$ & $P<0.00 I^{2}$ \\
History of dementia & & & $P=0.267^{\prime}$ \\
Source of referral & $34(36.6 \%)$ & $242(62.1 \%)$ & $P<0.0005^{\prime}$ \\
$\quad \begin{array}{l}\text { Medicine and allied } \\
\text { Surgery and allied }\end{array}$ & $59(63.4 \%)$ & $\mid 48(37.9 \%)$ & \\
\hline
\end{tabular}

Notes: 'Chi-squared tests; ${ }^{2}$ Two-tailed $t$-tests.

Abbreviation: SD, standard deviation.

surgical specialties (63.4\%) while nondelirious patients were mostly referred by the medical specialties (62.1\%). Taking into consideration that 24,171 patients were admitted to the surgical and allied departments and 24,073 patients were admitted to the medical and allied departments during the study year, the estimated annual prevalence of delirium in these departments were $0.24 \%$ and $0.14 \%$, respectively.

As shown in Table 2, the most common potential etiological factors that contributed to the development of delirium were fluid and electrolyte imbalance, fractures and infection, $(29.0 \%, 27.9 \%$, and $23.6 \%$, respectively). Thirty-four patients $(36.5 \%)$ had two or more potential etiological factors. Nine delirious patients (9.7\%) had a previous history of dementia as compared to 28 nondelirious patients $(7.2 \%)$; the difference was not statistically significant (Table 1).

\section{Management}

Medication had already been administered by the patients' physicians to 59 (63.4\%) of 93 delirious patients. Of those, $41(69.5 \%)$ had received haloperidol and the remaining 18 patients $(30.5 \%)$ some kind of benzodiazepines. Laboratory tests for the investigation of the disorder prior to the psychiatric assessment had been performed in only 12 patients (12.9\%). Table 3 shows the specific psychotropic medications administered by the CLPU staff. All the delirious patients were administered a kind of psychotropic drug, mainly antipsychotics $(86.0 \%)$, in contrast to nondelirious patients, of whom only $4.8 \%$ were prescribed antipsychotics. Delirious patients were treated mainly with first generation 
Table 2 Potential etiological factors of delirium

\begin{tabular}{lll}
\hline Precipitating factors & $\begin{array}{l}\text { Number of cases } \\
(\mathbf{n = 9 3 )}\end{array}$ & Percentage \\
\hline Fluid and electrolyte imbalance & 27 & 29.0 \\
Fractures & 26 & 27.9 \\
Infection & 22 & 23.6 \\
Drugs & 12 & 12.9 \\
Anemia & 9 & 9.6 \\
Alcohol withdrawal & 8 & 7.5 \\
Cancer & 7 & 7.5 \\
Myocardial infraction & 7 & 7.5 \\
Subdural hematoma & 7 & 7.5 \\
Organ failure & 6 & 6.4 \\
Stroke & 4 & 4.3 \\
Brain tumor & 3 & 3.2 \\
Other & 3 & 3.2
\end{tabular}

Note: The total number exceeds the number of cases because 34 patients (36.5\%) had two or more potential precipitating factors.

antipsychotics (73.7\%). Eight patients (8.6\%) were prescribed restraints, half of whom $(4.3 \%)$ were finally restrained according to the department's protocol, following written permission by the patients' relatives or law representatives. The relatives of the remaining four patients rejected the use of restraints and stated that their presence guaranteed the safety of the patient until the relief of the symptoms. None of the nondelirious patients was restrained.

\section{Impact of the delirium on CLPU service and length of hospitalization}

The delirious patients represented $19.2 \%$ of the total referrals. However, the number of evaluations conducted in delirious patients by CLPU staff during their hospitalization was much higher than that in nondelirious patients (3.13 vs 1.96 , respectively; $P<0.001$, Table 1 ), representing a percentage of $33.4 \%$ of the total evaluations. In 57 out of 93 delirious patients the duration of hospitalization was

Table 3 Psychotropic medications administered by CLPU staff

\begin{tabular}{cll}
\hline Psychotropic medication & $\begin{array}{l}\text { Delirious } \\
\text { patients } \\
(\mathbf{n}=\mathbf{9 3})\end{array}$ & $\begin{array}{l}\text { Nondelirious } \\
\text { patients } \\
(\mathbf{n}=\mathbf{3 9 0})\end{array}$ \\
\hline Antipsychotics & $80(86.0 \%)$ & $19(4.8 \%)$ \\
First generation antipsychotics & $59(73.7 \%)$ & $4(21.0 \%)$ \\
Second generation antipsychotics & $21(26.2 \%)$ & $15(78.9 \%)$ \\
Benzodiazepines & $13(13.9 \%)$ & $26(6.7 \%)$ \\
\hline
\end{tabular}

Abbreviation: CLPU, Consultation-Liaison Psychiatry Unit. prolonged from three to eight days because of the delirium, as estimated by the difference between the actual discharge date and the scheduled discharge date prior to the onset of the delirium.

\section{Discussion Referrals, diagnoses, and rates}

The results of the present study showed that, in our hospital, the annual psychiatric referral rate was $1 \%$. Several studies have demonstrated that the annual psychiatric referral rates range from $1.3 \%$ to $5.8 \% .^{23-27}$ Although the referral rate in our hospital is lower than the rates reported by other studies, one must take into consideration that our CLPU has been recently established, and that the number of referrals increased the following year, presumably as a result of our unit's development and educational activities within the hospital. ${ }^{21}$

Diagnosis of delirium was difficult or impossible in a remarkable number of patients that were referred for "delirium" (7.96\%), due to delayed referral. Retrospective examination revealed that seven of these nine patients had developed delirium; the majority were aged over 80 years, with the hypoactive type of the syndrome and a history of dementia. This is consistent with the results of previous studies, ${ }^{17}$ which have demonstrated that older age, history of dementia and hypoactive delirium constitute risk factors for nonrecognition of delirium and, consequently, delayed referral.

In 11 of 113 patients $(9.73 \%)$ the final diagnosis was different from "delirium," despite the fact that this was the reason for the referral. The prominent symptom that prompted such referrals was "agitation", but these patients were eventually diagnosed as having depression, dementia or psychosis. This is in line with the results of previous studies that have pointed out that "disruptive behavior" is a major reason for referral, ${ }^{17,23}$ but also indicates that, in some cases, the hospital staff failed to fully recognize the underlying cause of agitation, and consequently delirium. One additional factor that possibly contributes to the misdiagnosis and mistreatment of delirium is, in our opinion, the various terms used for the disorder in Greek (eg, delirium, organic psychosyndrome, paralerema [a word often used for delusions in Greek], or intensive care unit syndrome) which hinders communication across different medical specialties.

\section{Patients' profile}

Our findings showed that patients with delirium were older than patients in the nondelirious group and that $76.3 \%$ were 
aged over 70 years. This is in line with most previous studies which report that delirium is more frequent in older ages ${ }^{23,28}$ and further confirms the comprehensive recommendations of "delirium guidelines for general hospitals,"6 which suggest that an age $>70$ years is regarded as a serious (code A) predisposing factor on admission. Our results also showed an overrepresentation of males among delirious patients (60\%) versus nondelirious patients (46\%), a finding similar to Grover and colleagues, ${ }^{23}$ which could be attributed to the fact that hyperactive delirious male patients may be more agitated and difficult to be controlled than their female counterparts, thus triggering a referral.

In our study delirious patients were mostly referred to the CLPU by the surgical specialties $(63.4 \%)$, in contrast to nondelirious patients who were mostly referred by medical specialties $(62.1 \%)$. This could be attributed to the high prevalence of the disorder among surgical populations, ${ }^{3}$ but it may also mean that the medical specialties are more acquainted with the recognition and management of delirium.

Common etiological factors associated with delirium were fluid and electrolyte imbalance (29\%), fractures (28\%), and infections (24\%), with $36 \%$ of the cases having two or more causes, findings which are compatible with current knowledge about the etiology of delirium. ${ }^{28}$ A preexisting history of dementia was evident in $9.7 \%$ of the referred delirious patients, which is much lower than the rates cited by other studies, ${ }^{12,17,29}$ given also that it is estimated that up to two thirds of cases of delirium occur superimposed on dementia. ${ }^{30-32}$ This could be attributed to the difficulties that medical staff face in the recognition of delirium in patients suffering from dementia, possibly attributing the syndrome's symptoms to the existing dementia. ${ }^{17}$ Therefore, CLPU services may have an additional role in this by offering education, training and advice to staff in distinguishing delirium from worsening dementia, a task that can be particularly difficult.

\section{Management}

Our findings showed that a remarkable number of patients $(63.4 \%)$ had been administered medications by their physicians prior to the referral. Despite this, psychiatric consultation was requested due to the following factors: 1) continuation of the symptoms (which could be attributed to the natural course of the syndrome, the inadequate dose of the medication or the inappropriate medication, ie, benzodiazepines instead of antipsychotics), and 2) the need for an experienced opinion to verify the diagnosis and confirm the appropriate administration of the medication.
The small number of delirium cases that underwent laboratory examinations for the investigation of the predisposing factors by their physicians, prior to the referral, $(12.9 \%)$ is rather surprising, indicating that delirium is often overlooked by the clinicians caring for the patient. This further emphasizes the aforementioned under-recognition of delirium and, as already has been mentioned, ${ }^{30}$ possibly reflects the lack of appreciation of delirium as a potential medical emergency and the prevailing belief that delirium is a disorder with vague etiology and symptomatic treatment.

Patients diagnosed with delirium by the CLPU staff were mainly administered first generation antipsychotics, which historically have been used in the treatment of the syndrome, although the use of a second generation antipsychotic in $26 \%$ of the cases indicates the changing trend, since evidence suggests that second generation antipsychotics are preferred in some cases because of their safer profile. ${ }^{33}$

Patients displaying delirium sometimes require the use of prophylactic measures, such as restraints, although restraints themselves are considered as a risk factor for delirium. ${ }^{6}$ In our sample, eight delirious patients required restraint, although only four had been restrained following written permission by their relatives. The remaining four patients were eventually not restrained, because their families did not consent to the use of restraints, offering their availability to supervise the patient on a 24-hour basis. This involvement resulted in less use of restraints without the need of higher doses of antipsychotics to these four patients, indicating that the role of the family is important in the care of the patient. Thus it could be suggested that appropriate education of the family regarding the disorder could lead to a more favorable course and outcome.

\section{Impact of delirium on CLPU service and length of hospitalization}

Our findings revealed that the number of the evaluations conducted in delirious patients by CLPU staff was much higher than that in nondelirious patients, indicating that the CLPU staff dedicated a significant proportion of their time to the care of the delirious patients. It has been reported that delirium is associated with greater use of hospital resources, ${ }^{9-11}$ and this, along with our findings, underlines the need for proper CLPU service organization in order to be able to meet the increased needs of delirious patients. Besides, involvement of CLPU psychiatrists in the care of these patients improves the cost-benefit aspect of CLPU's psychiatry service through accurate diagnoses, prompt treatments, and shortened hospital stays, while it has also 
been reported that involvement of CLPU psychiatrists in the care of delirious patients alleviates patient and family distress during the course of the syndrome ${ }^{18}$ and would justify the use of their services. ${ }^{17}$

In accordance with the results of previous studies, ${ }^{10,34-39}$ our findings also showed that the estimated duration of hospitalization was prolonged due to delirium in a significant proportion of delirious patients. Some studies, however, did not find the same association. ${ }^{38,40}$ Our findings support the need for early intervention and prevention of delirium, as has been stressed by previous studies. ${ }^{41}$ Taking also into consideration the physicians' difficulty in recognizing delirium, further staff educational activities provided by the CLPU unit are needed.

\section{Strengths and limitations}

There were a number of limitations of this study, which need to be recognized. Different psychiatric consultants provided services, and standardized psychiatric scales and structured clinical interviews for the diagnosis of delirium were not used. This study also does not examine the characteristics of the referring service or the perceived quality of the consultation, which may influence the decision to refer. On the other hand, the strengths of this study were the use of our electronic database and the fact that our sample could be regarded a representative sample of the referrals, since our unit receives all the hospital referrals.

\section{Conclusion}

Our study showed that referrals for delirium are rather frequent in a CLPU in Greece. It also appears that although delirium is a common condition in general hospitals, it remains a 'confusing' condition for health practitioners. The under-recognition of deliria, the estimated prolonged hospitalization and the time that our unit dedicated to the delirious patients underlines the role of the CLPU psychiatrists in the management of delirium, which is not only to treat the patients' delirium but also to inform and educate the physicians and hospital staff of other disciplines regarding the management of the syndrome. Besides, as Kornfeld has pointed out, ${ }^{42}$ CLPU psychiatry or psychosomatic medicine should survive and flourish because the substantial presence of this subspecialty means better medical care through the direct clinical work of its practitioners as well as their teaching and research.

\section{Disclosures}

The authors have no conflicts of interest.

\section{References}

1. Leentjens AF, MacLullich AM, Meagher DJ. Delirium, Cinderella no more...? J Psychosom Res. 2008;65:205.

2. American Psychiatric Association. Diagnostic and Statistical Manual of Mental disorders. 4th ed. Washington, DC: American Psychiatric Association; 2000.

3. Bucht G, Gustafson Y, Sandberg O. Epidemiology of delirium. Dement Geriatr Cogn Disord. 1999;10:315-318.

4. Lindesay J, Rockwood K, Rolfson DB. The epidemiology of delirium. In: Lindesay J, Rockwood K, Macdonald AJ, editors. Delirium in Old Age. New York, NY: Oxford University Press; 2002. p. 27-50.

5. Elie M, Rousseau F, Cole M, Primeau F, McCusker J, Bellavance F. Prevalence and detection of delirium in elderly emergency department patients. CMAJ. 2000;163:977-981.

6. Michaud L, Büla C, Berney A, et al. Delirium Guidelines Development Group. Delirium: guidelines for general hospitals. J Psychosom Res. 2007;62:371-383.

7. Inouye SK, Bogardus ST, Charpentier PA, et al. A multicomponent intervention to prevent delirium in hospitalized older patients. $N$ Engl J Med. 1999;340:669-676.

8. Inouye SK. Delirium in older persons. $N$ Engl J Med. 2006;354: $1157-1165$.

9. Cole MG, Primeau FJ, Elie LM. Delirium: prevention, treatment, and outcome studies. J Geriatr Psychiatry Neurol. 1998;11:126-137.

10. Pompei P, Foreman M, Rudberg MA, Inouye SK, Braund V, Cassel CK. Delirium in hospitalized older patients: outcomes and predictors. J Am Geriatr Soc. 1994;41:809-815.

11. Maguire GP, Julier DL, Hawton KE, Bancroft JH. Psychiatric morbidity and referral on two general medical wards. BMJ. 1974;1:268-270.

12. Holden J, Jayathissa S, Young G. Delirium among elderly general medical patients in a New Zealand hospital. Intern Med J. 2008;38: 629-634.

13. Hustey FM, Meldon SW. The prevalence and documentation of impaired mental status in elderly emergency department patients. Ann Emerg Med. 2002;39:248-253.

14. Morrison RS, Magaziner J, Gilbert M, et al. Relationship between pain and opioid analgesics on the development of delirium following hip fracture. J Gerontol A Biol Sci Med Sci. 2003;58:76-81.

15. Farrell KR, Ganzini L. Misdiagnosing delirium as depression in medically ill elderly patients. Arch Intern Med. 1995;155: 2459-2464.

16. Leentjens AF, Diefenbacher A. A survey of delirium guidelines in Europe. J Psychosom Res. 2006;61:123-128.

17. Mittal D, Majithia D, Kennedy R, Rhudy J. Differences in characteristics and outcome of delirium as based on referral patterns. Psychosomatics. 2006; 47:367-375

18. Lipowski ZJ. Delirium: Acute confusional states. New York, NY: Oxford University Press; 1990.

19. Gournas G, Madianos MG, Stefanis CN. Psychological functioning and psychiatric morbidity in an elderly urban population in Greece. Eur Arch Psychiatry Clin Neurosci. 1992;242(2-3):127-134.

20. Papaioannou A, Fraidakis O, Michaloudis D, Balalis C, Askitopoulou $\mathrm{H}$. The impact of the type of anaesthesia on cognitive status and delirium during the first postoperative days in elderly patients. Eur $J$ Anaesthesiol. 2005;22:492-499.

21. Hyphantis T, Mantas C, Mavreas V. The psychodynamically oriented consultation-liaison psychiatry unit, University of Ioannina, Greece. J Psychosom Res. 2009;66:95-97.

22. Altman DG. Practical Statistics for Medical Research. London, UK: Chapman and Hall; 1991.

23. Grover S, Subodh BN, Avasthi A, et al. Prevalence and clinical profile of delirium: a study from a tertiary-care hospital in north India. Gen Hosp Psychiatry. 2009;31:25-29.

24. Hales RE, Polly S, Bridenbaugh H, Orman D. Psychiatric consultations in a military hospital: a report on 1065 cases. Gen Hosp Psychiatry. 1986;8:173-182. 
25. Clarke DM, Smith GC. Consultation-liaison psychiatry in general medical units. Aust N Z J Psychiatry. 1995;29:424-432.

26. Bourgeois JA, Wegelin JA, Servis ME, Hales RE. Psychiatric diagnoses of 901 inpatients seen by consultation-liaison psychiatrists at an academic medical center in a managed care environment. Psychosomatics. 2005;46:47-57.

27. Grant JE, Meller W, Urevig B. Changes in psychiatric consultations over ten years. Gen Hosp Psychiatry. 2001;23:261-265.

28. Levkoff S, Cleary P, Liptzin B, Evans D. Epidemiology of delirium: an overview of research issues and findings. Int Psychogeriatr. 1991;3:149-167.

29. Laurila JV, Laakkonen ML, Tilvis RS, Pitkala KH. Predisposing and precipitating factors for delirium in a frail geriatric population. J Psychosom Res. 2008;65:249-254.

30. Inouye SK. The dilemma of delirium: clinical and research controversies regarding diagnosis and evaluation of delirium in hospitalized elderly medical patients. Am J Med. 1994;97:278-288.

31. Levkoff SE, Besdine RW, Wetle T. Acute confusional states (Delirium) in the hospitalized elderly. Ann Rev Gerontol Genatr. 1986;6:1-26.

32. Francis J, Kapoor WN. Delirium in hospitalized elderly. J Gen Intern Med. 1990;5:65-79.

33. Bourne RS, Tahir TA, Borthwick M, Sampson EL. Drug treatment of delirium: past, present and future. J Psychosom Res. 2008;65:273-282.
34. Francis J, Martin D, Kapoor WN. A prospective study of delirium in hospitalized elderly. JAMA. 1990;8:1097-1101.

35. O'Keeffe S, Lavan J. The prognostic significance of delirium in older hospital patients. J Am Geriatr Soc. 1997;45:174-178.

36. Thomas RI, Cameron DJ, Fahs MC. A prospective study of delirium and prolonged hospital stay. Arch Gen Psychiatry. 1988;45:937-940.

37. George J, Bleasdale S, Singleton SJ. Causes and prognosis of delirium in elderly patients admitted to a district general hospital. Age Ageing. 1997;26:423-427.

38. McCusker J, Cole MG, Dendukuri N, Belzile E. Does delirium increase hospital stay? J Am Geriatr Soc. 2003;51:1539-1546.

39. Ely EW, Gautam S, Margolin R, et al. The impact of delirium in the intensive-care unit on hospital length of stay. Intensive Care Med. 2001;27:1982-1900.

40. Inouye SK, Rushing JT, Foreman MD, Palmer RM, Pompei P. Does delirium contribute to poor hospital outcomes? A three-site epidemiologic study. J Gen Intern Med. 1998;13:234-242.

41. Young J, Leentjens AF, George J, Olofsson B, Gustafson Y. Systematic approaches to the prevention and management of patients with delirium. J Psychosom Res. 2008;65:267-272.

42. Kornfeld DS. Consultation-liaison psychiatry: contributions to medical practice. Am J Psychiatry. 2002;159:1964-1972.
International Journal of General Medicine

\section{Publish your work in this journal}

The International Journal of General Medicine is an international, peer-reviewed open-access journal that focuses on general and internal medicine, pathogenesis, epidemiology, diagnosis, monitoring and treatment protocols. The journal is characterized by the rapid reporting of reviews, original research and clinical studies across all disease areas.

\section{Dovepress}

A key focus is the elucidation of disease processes and management protocols resulting in improved outcomes for the patient.The manuscript management system is completely online and includes a very quick and fair peer-review system. Visit http://www.dovepress.com/ testimonials.php to read real quotes from published authors. 